\title{
躍Irestice
}

\section{Validação do jogo digital PREVINIX para prevenção de Infecções Sexualmente Transmissíveis na adolescência}

\author{
Validation of the PREVINIX digital game for the prevention of Sexually Transmitted \\ Infections in adolescence
}

\begin{abstract}
Silvia Roberta Peixoto Silva de Oliveira ${ }^{1}$, Danielle de Oliveira Silva Valérioº, Marina Araújo Rosas $^{3}$, Larissa Negromonte Azevedo ${ }^{4}$, Beatriz do Nascimento Silva ${ }^{5}$, Daniela Tavares Gontijo 6
\end{abstract}

\section{Resumo}

Introdução: A adolescência caracteriza-se como um período de vulnerabilidade para contaminação pela Infecções Sexualmente Transmissiveis (IST's), sendo de vital importância o desenvolvimento de tecnologias educativas direcionadas para esta temática. Objetivo: validar o jogo Previnix para a construção de conhecimentos sobre a prevenção, identificação e tratamento de IST'S na adolescência. Métodos: Pesquisa de validação realizada em escola pública do Recife. Participaram 56 adolescentes (12-16 anos). Os dados foram coletados através de questionários (antes e após), gravação da ação educativa, formulário e grupo focal. Os dados quantitativos foram descritos termos de acertos e erros ou através do Indice de Concordância (IC) e os dados qualitativos submetidos a análise temática. O Previnix alcançou IC total de $91 \%$ e pontuação superior a $80 \%$ de concordância em todos os itens avaliados. Houve aumento do número de acertos nos questionários após a intervenção e realizadas sugestões para aprimoramento do jogo. A utilização da tecnologia possibilitou a construção de conhecimentos de forma dialógica. Conclusão: O Previnix foi validado como tecnologia educativa para utilização em ações de promoção saúde sexual e reprodutiva com adolescentes.

Palavras-Chave: Adolescência; Educação em Saúde; doenças sexualmente transmissíveis; Saúde sexual e reprodutiva.

\begin{abstract}
Introduction: Adolescence is characterized as a period of vulnerability to contamination by sexually transmitted infections(STI), and the development of educational technologies directed to this theme is vitally important. Objective: validate the game Previnix that is directed towards the knowledge construction about prevention, identification and treatment of STI in adolescence. Methods: Methodological research of validation carried out in a public school in Recife. Participants were 56 adolescents (12 -16 anos). The data were collected through questionnaires (before and after) about the infections, recording of the educational section, form approaching objective, relevance, structure and presentation of the game and focus group. The quantitative data resulted in the elaboration of the Concordance Index and
\end{abstract}

1 Terapeuta Ocupacional. Universidade Federal de Pernambuco. Email: silviarpeixoto@ymail.com

2 Terapeuta Ocupacional. Universidade Federal de Pernambuco. Email: danielleoliveiras@outlook.com

3 Terapeuta Ocupacional. Docente da Universidade Federal de Pernambuco. Email: marinaarosas@gmail.com

4 Médica Infectologista. Docente Centro Universitário de João Pessoa. Email: Iarissanegromonte@gmail.com

5 Graduanda em Terapia Ocupacional. Universidade Federal de Pernambuco. Email: eubeanascimento@gmail.com

6 Terapeuta Ocupacional. Docente da Universidade Federal de Pernambuco. Email: danielatgontijo@gmail.com

Correspondência: Rua Coronel Waldemar Basgal, 393 - Piedade Jaboatão dos Guararapes - PE, Brasil. CEP 54400-170.

RE. SAÚD. DIGI. TEC. EDU., Fortaleza, CE, v.4, n.2, p.163-178, ago./dez. 2019.

ISSN: 2525-9563 
the qualitative ones were submitted to the thematic analysis. Previnix achieved a total $\mathrm{Cl}$ of $91 \%$ and scores higher than $80 \%$ of concordance in all items evaluated. There was an increase in the number of correct answers in the questionnaires after the intervention and suggestions for improvement of the game. It was observed that the use of technology made possible the construction of knowledge in a dialogical way. Conclusion: Previnix was validated as an educational technology for use in sexual and reproductive health promotion actions with adolescents.

Keywords: Adolescence; Health Education; Sexually Transmitted Diseases; sexual and reproductive health.

\section{Introdução}

A adolescência é uma etapa do desenvolvimento que precisa ser compreendida a partir das mudanças biopsicossociais. Entre estas se destaca a vivência das primeiras experiências sexuais ${ }^{1}$. Considerando a descoberta e vivência da sexualidade como aspecto importante no adolescer assim como os tabus, preconceitos e mitos relacionados a esta experiência, os e as adolescentes constituem um grupo que apresenta grande vulnerabilidade às Infecções Sexualmente Transmissíveis (IST's) ${ }^{2}$.

Pesquisa Nacional de Saúde do Escolar $^{5}$ apontou que $27,5 \%$ dos adolescentes que frequentam o 9ำ ano já tiveram relação sexual alguma vez, sendo que somente $61,2 \%$ relataram ter usado preservativo na primeira relação sexual. Observa-se também que nas relações consideradas estáveis pelos jovens, em somente $40 \%$ há o uso de preservativo ${ }^{3}$. Revisão integrativa que buscou identificar as causas da não utilização de preservativos entre adolescentes

encontrou a dificuldade na negociação do uso como um dos principais fatores ${ }^{4}$. Além disso, também se inter-relacionam na explicação desta atitude a sensação de confiança no parceiro, desconhecimento sobre o preservativo e as IST's, não gostar do uso, desejo de engravidar, orientação inadequada e uso de álcool e outros drogas ${ }^{4}$.

A não utilização dos preservativos refletem-se nos altos índices de IST's na adolescência ${ }^{5}$.

$\mathrm{Na}$ perspectiva da promoção da saúde integral e considerando a vivência da sexualidade de forma segura como um direito do adolescente, o desenvolvimento de estratégias de diminuição da vulnerabilidade as IST's e o aumento da prática do sexo seguro constitui-se como uma das prioridades para a Atenção Básica².

Neste contexto a educação em saúde se constitui como ferramenta efetiva de interlocução com os jovens em diferentes espaços sociais, incluindo os serviços de saúde e educação. As ações de educação em saúde devem contribuir para o desenvolvimento do protagonismo 
adolescente frente ao seu processo de saúde e bem estar². Para isto, o uso de metodologias que estimulem não só a participação, mas sobretudo posturas críticas do adolescente podem ter maior potencial de efetivação para a promoção de mudanças reais no cotidiano ${ }^{2,6}$. Estas ações educativas podem ser mediadas por diferentes instrumentos, entre eles os jogos educativos digitais ${ }^{6}$. No campo da promoção da saúde sexual e reprodutiva os jogos digitais têm sido alvo de estudo em âmbito internacional e recentemente também no cenário nacional ${ }^{6,7,8,9,10,11}$.

Estudos apontam que os jogos digitais atraem a participação dos adolescentes, favorecem a discussão da temática, marcada ainda como tabu social, de forma mais leve e divertida, estimulam a interatividade e o exercício de processos de tomada de decisão, além de contribuírem para a construção de conhecimentos que podem subsidiar a escolha por atitudes promotoras de saúde $^{9,10}$. Estes estudos enfatizam a importância de desenvolvimento de outros jogos para a abordagem de temáticas relacionadas à sexualidade na adolescência concomitante a avaliação sistemática destes enquanto tecnologias educativas.

Neste estudo objetivou-se validar, com adolescentes, o jogo digital PREVINIX enquanto tecnologia educativa direcionada para a produção de conhecimentos sobre a prevenção, identificação e tratamento de IST's na adolescência. Especificamente objetivou-se descrever as informações sobre IST's antes e após a utilização do jogo; validar o jogo nos domínios objetivo, relevância, estrutura e apresentação; analisar a vivência educativa e identificar as sugestões para aprimoramento da tecnologia educativa.

\section{PREVINIX}

O jogo digital PREVINIX é uma tecnologia educativa direcionada para a promoção da saúde sexual e reprodutiva de adolescentes, que objetiva, especificamente, construir conhecimentos com adolescentes sobre a identificação, prevenção e tratamento das IST's. O conteúdo do jogo foi selecionado considerando dados epidemiológicos sobre as IST's na adolescência ${ }^{5}$ em diálogo com os questionamentos e dúvidas colhidas durante experiências de extensão e pesquisa desenvolvidas pelo Núcleo de Estudos e Pesquisas em Vulnerabilidade e Saúde na Infância e Adolescência (NEPVIAS-UFPE) $^{1,10,12}$. Destaca-se que a versão física do PREVINIX (que se constitui como um jogo de roleta tradicional) já é utilizado e avaliado permanentemente, no que se refere ao seu conteúdo, nas ações de educação em saúde pelo NEPVIAS desde $2013^{12}$.

O jogo foi desenvolvido para ser utilizado com adolescentes entre 12 a 18 anos. O PREVINIX apresenta como diferencial, em relação aos outros jogos digitais educativos comumente 
disponíveis, o fato de ter sido elaborado para ser jogado por grupos de adolescentes mediados por um profissional de saúde ou educação. Assim, o jogo é operacionalizado em um computador e projetado para visualização coletiva. O cenário do jogo é constituído por um tabuleiro, roleta e pinos (FIGURA 1).

Figura 1: Cenário do jogo Previnix.

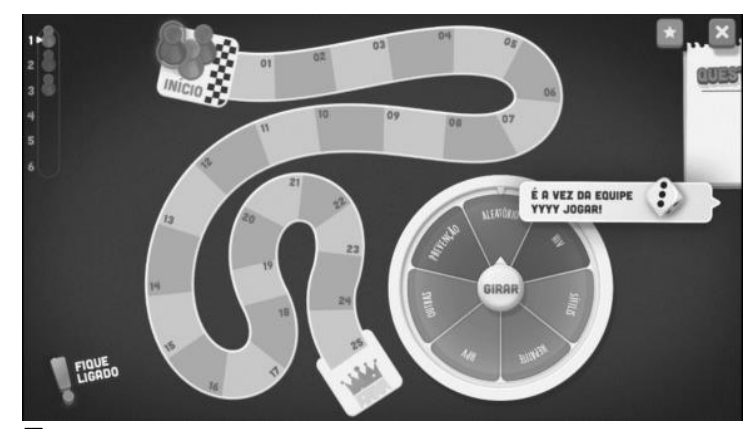

Fonte: os autores

Para jogar, os adolescentes são divididos em grupos que correspondem às cores dos pinos. Durante o jogo, os grupos sucessivamente rodam a roleta, constituída por 7 espaços que correspondem a 6 temas específicos e 1 aleatório. Os temas se referem a medidas de prevenção gerais relacionadas ao sexo seguro e também as IST's: HIVIAIDS, HPV, Sífilis, Hepatites virais, Clamídia, Gonorréia e Tricomoníase (estes 3 últimos constituem o mesmo espaço na roleta). Cada temática contém afirmativas que deverão ser respondidas como verdadeiras ou falsas, e abordam formas de transmissão, sinais e sintomas, tratamento e prevenção.
O PREVINIX foi desenvolvido para mediar ações educativas que se caracterizem pela construção conjunta de conhecimentos entre educadores e adolescentes ${ }^{14}$. Assim, durante a utilização do jogo cabe ao educador oportunizar que os adolescentes expressem seus conhecimentos prévios sobre os temas em discussão e juntos com eles construa novos conhecimentos que tenham significado e repercussão em seus cenários reais de vida. Além disso, espera-se que o educador problematize cada afirmativa com os participantes antes das respostas disponíveis no jogo, assim como as escolhas realizadas pelos mesmos.

Após a problematização da afirmativa pelo educador com os e as adolescentes participantes, a opção é escolhida e a resposta correta aparece na tela juntamente com uma explicação ou justificativa que também é explorada pelo responsável pela condução da ação. Se acertarem a questão, os grupos avançam no tabuleiro. Durante o jogo, em algumas casas há prendas surpresa que caso o grupo opte por realizar poderão avançar mais uma casa. No jogo os grupos se revezam e ganha quem alcançar a linha de chegada primeiro. $\mathrm{O}$ jogo ainda oferece ao educador a possibilidade de exploração de imagens ilustrativas do conteúdo.

A versão validada do Previnix, assim como o material de apoio que subsidia sua utilização podem ser solicitados 
gratuitamente pelo email: nepviasufpe@gmail.com.

\section{Métodos}

Estudo de validação de tecnologia do tipo pesquisa metodológica, com análise de dados quantitativos e qualitativos. Foram convidados a participar do estudo adolescentes com idades entre 12 e 18 anos de uma escola pública da cidade do Recife, matriculados no $8^{\circ}$ ano do ensino fundamental.

Os dados foram coletados em três momentos: antes da ação educativa com o PREVINIX, durante e após o término desta. Antes da utilização do jogo, aplicouse um questionário, formulado pelas pesquisadoras, contendo 25 afirmativas relacionadas às formas de transmissão, sinais e sintomas, tratamento e prevenção das IST's, nas quais os adolescentes responderam se concordavam, discordavam ou não sabiam.

Durante a ação educativa, o jogo foi conduzido pelas pesquisadoras, sendo a experiência gravada em equipamento digital e posteriormente transcrita. Após a ação educativa, os adolescentes responderam novamente ao questionário sobre as IST's e preencheram um formulário com questões relacionadas aos objetivos, relevância, estrutura e apresentação do jogo. Este formulário, elaborado com base no estudo de Monteiro $^{12}$ continha afirmativas com respostas em uma escala Likert (Concordo totalmente; Concordo parcialmente; Nem concordo nem discordo; Discordo parcialmente e Discordo totalmente). No mesmo formulário também havia espaço para que pudessem ser realizadas sugestões e observações sobre o jogo.

Além disso, os jovens participaram de um grupo focal, que foi gravado e posteriormente transcrito, conduzido com base em um roteiro semiestruturado que abordou aspectos referentes vivência da experiência educativa.

Os dados dos questionários aplicados antes após a ação educativa foram descritos em termos de número de acertos e erros.

Os dados dos formulários, aplicados após a ação educativa, foram analisados através de um índice de concordância (IC). A elaboração de índices de concordância é utilizada em estudos de validação com público alvo ${ }^{15}$ e se referem a concordância dos participantes em relação a resposta positiva. Neste estudo, considerou-se como resposta positiva concordo totalmente ou parcialmente e optou-se por elaborar: a) IC por item - a partir da soma das respostas positivas (concordo totalmente e concordo parcialmente); b) IC por domínio - avaliado através da soma das médias dos ICs por item; c) IC geral calculado através da média aritmética dos ICs dos domínios. Seguindo recomendações para estudos metodológicos que de validação foi considerado como ponto de corte IC de $80 \%(0,80)$. 
Os dados qualitativos provenientes foram submetidos à análise de conteúdo e agrupados em categorias que refletem os domínios de validação (objetivo, relevância, estrutura e apresentação) e a vivência da experiência educativa. Os e as adolescentes são identificados como AF para sexo feminino e AM para sexo masculino.

A pesquisa foi aprovada pelo Comitê de Ética e Pesquisa (CEP) de acordo com parecer 116863/2017. A participação dos adolescentes foi condicionada à assinatura do Termo de Consentimento Livre e Esclarecido (TCLE) pelos seus responsáveis legais, e do Termo de Assentimento Livre e Esclarecido (TALE) pelos próprios adolescentes.

\section{Resultados}

O processo de validação foi realizado em 4 ações educativas (4 grupos), nas quais participaram, no total, 56 adolescentes ( 35 do sexo feminino e 21 do sexo masculino), com idade média 12,9 anos e mediana 13 anos, sendo 12 anos a menor idade e 16 anos a maior.

Os resultados da pesquisa serão apresentados no que se refere a descrição do conhecimento sobre IST's antes e após a utilização do jogo (dados obtidos com os questionários), aos dados referentes aos domínios de validação (obtidos através do formulário e das transcrições das ações educativas e grupos focais) e à vivência da ação educativa mediada pelo PREVINIX (dados provenientes dos grupos focais).

\section{a) Conhecimento sobre IST's antes e após a utilização do jogo}

A análise do questionário sobre as IST's apontou que a experiência educativa com o PREVINIX possibilitou ganho de informações, uma vez que se observou, quando se considera todas as temáticas, aumento de cerca de $100 \%$ no número de acertos, com expressiva diminuição das respostas "não sei" após a ação (Quadro 1).

Nota-se que a diminuição do "Não sei" além de estar relacionada com o aumento das alternativas corretas, foi concomitante a um pequeno aumento (5,9\%) das alternativas incorretas, o quê pode caracterizar maior disponibilidade dos jovens em se arriscarem a responder questões sobre temas antes desconhecidos. Essa interpretação foi corroborada pelos relatos dos adolescentes que ao preencherem novamente 0 questionário enfatizaram: "Grupo D) AM: Minhas respostas mudaram demais!

Considerando as temáticas específicas observou-se que as questões sobre HIV/AIDS obtiveram maior número de acertos antes da ação educativa quando comparadas com outros temas, demonstrando mais informações prévias dos adolescentes sobre o assunto.

A análise do questionário também apontou maior ganho de informações em relação à clamídia/gonorréia/tricomoníase e sífilis, com aumento de $65,2 \%$ e $48,3 \%$ 
do número de acertos após o jogo, respectivamente. Nas afirmativas referentes à sífilis, destaca-se que 0 quantitativo de "Não sei", antes do jogo, apresentou um percentual de 66,1\%, diminuindo para 9,3\% após a ação educativa.

\section{b) Resultados referentes aos domínios de validação}

A análise do formulário apontou que o jogo foi validado pelos adolescentes como uma tecnologia educativa no campo em discussão, uma vez que alcançou um IC geral de $91 \%$ e pontuação superior a $80 \%$ de concordância em todos os itens avaliados.

\section{b-1)Domínio Objetivo}

No domínio Objetivo, que se refere a avaliação se os propósitos, metas ou fins foram atingidos pelo jogo, foi obtido um IC de 93\% (Quadro 2). A análise dos dados qualitativos possibilitou a constatação da materialização dos objetivos do PREVENIX uma vez que se observou situações nas quais os adolescentes participam ativamente das discussões, destacando-se as que se constroem em torno do tema da prevenção.

(Grupo A) AF: Pode usar a camisinha masculina e feminina ao mesmo tempo? (Grupo A) AM: oxi, tem camisinha pra mulher?(...)

(Grupo B) AM:Borracha com borracha vai rasgar!
Destaca-se que na ação educativa com o PREVINIX também oportunizou-se a construção de discussões sobre a identificação das IST's, a importância de se observar o corpo para identificação de sinais e sintomas destas além de reflexões sobre as suas consequências.

Por fim, em relação ao domínio objetivo, congruente ao dado quantitativo que apontou o menor índice de concordância (89\%), os adolescentes apesar de compreenderem que 0 PREVINIX contribui para a busca da rede de suporte para o tratamento e para 0 conhecimento de como este deve ser feito, sugeriram no grupo focal, a inclusão de mais perguntas sobre este assunto no jogo.

\section{b.2) Domínio Relevância}

Neste domínio foi avaliado se os temas do jogo são significativos para os adolescentes possibilitando a construção de informações e aprendizado, e se o mesmo é considerado importante no contexto da vida. Neste domínio, o índice de concordância obtido foi de $91 \%$ validando todos os itens (Quadro3).

A questão da relevância temática do PREVINIX foi alvo de discussão nos grupos focais, sendo que os participantes referiram que é um assunto constrangedor, mas muito importante para ser tratado, pois muitos não sabem como lidar diante das situações de risco. 
(Grupo A) AM: Eu acho muito engraçado (...)

(Grupo A) AM: não é engraçado, é constrangedor!

(Grupo A) AM: Sim, porque acontece do adolescente nem saber que ta correndo perigo

Os participantes relataram que a ação educativa mediada pelo PREVINIX permitiu a descoberta de infecções ainda desconhecidas, aquisição de conhecimento e informações acerca do uso do preservativo, de como se prevenir, sinais e sintomas e formas de tratamento. Tais afirmações corroboram os dados já apresentados no domínio objetivo e também apresentados em relação ao questionário aplicado antes e depois.

\section{c) Domínio Estrutura e Apresentação}

Neste domínio são elencadas questões que se referem a se o jogo é apropriado para ser utilizado com os adolescentes, se as afirmações e imagens estavam de fácil compreensão e se era atrativo. Neste domínio, o índice de concordância obtido foi de $91 \%$ (quadro 4).

No grupo focal, os adolescentes relataram que as afirmativas do jogo são de fácil compreensão e muito claras. Uma adolescente sugeriu que não fossem utilizadas abreviações nas frases do jogo para que todos pudessem compreender melhor.
No que se refere a apresentação gráfica e o nome do jogo, os dados das transcrições das ações apontaram que o recurso é facilmente reconhecido como um jogo e que o nome PREVINIX é adequado à temática foco do mesmo.

Pesquisadora: Agora com esse nome aqui, Previnix. Vocês acham que é um jogo que fala sobre o que?

(Grupo B) AF: Prevenção de doenças?

(Grupo D) AM: Um jogo de doenças sexualmente transmissíveis

Um ponto que suscitou discussões nos grupos focais foi em relação as imagens ilustrativas das IST's no jogo. A maioria dos participantes inicialmente relatou que não gostaram destas, pois acharam chocantes, mas ao mesmo tempo concordam que são importantes por mostrarem a realidade e ajudarem a entender melhor como cada IST se manifesta no corpo do indivíduo. Alguns participantes sugeriram que fossem utilizadas imagens de estágios iniciais das doenças.

(Grupo A) AM: Não, professora! Achei nojento algumas

Pesquisadora: Mas vocês acharam importante ter essas imagens? Ou deveríamos tirar?

(Grupo A) AM: Não tira!

(Grupo A) AM: São importantes! Só ameniza

(Grupo B) AF: Não porque mostra a realidade, dá pra entender melhor 
Ainda em relação a estrutura do jogo, os adolescentes sugeriram que fossem retiradas as prendas que fazem parte do jogo ou que as mesmas fossem mais variadas, uma vez que se constituem principalmente pela solicitação de cantar músicas. Além disso foi sugerido a diminuição do tempo disponível para a resposta as questões pelos grupos.

\section{d) Análise da Vivência da experiência}

\section{educativa}

Nos grupos focais os jovens foram convidados a refletiram sobre a vivência da experiência de aprender através de um jogo, sendo destacado por estes que esta estratégia caracteriza-se como uma forma de aprendizagem mais efetiva, atrativa e divertida diferente das utilizadas tradicionalmente nas salas de aula.

(Grupo C) AF: É melhor do que nossas aulas

(Grupo D) AF: O que ruim é que aqui a gente não ganha ponto, lá em cima (sala de aula) respondeu ganha ponto

Pesquisadora: $E$ aqui vocês ganham 0 que?

(Grupo D) AF: Conhecimento. Melhor do que ponto

(Grupo C) AM: É diferente. Assim é mais aberto do que na sala, é melhor pra conversar.

Os jovens afirmaram que com o uso do PREVINIX puderam conversar mais abertamente sobre os assuntos e compreendem que isso potencializa a aprendizagem de forma coletiva, podendo o recurso ser utilizados por profissionais de saúde e educação. Destaca-se que os participantes sugerem que o jogo seja disponibilizado para uso em smartphone para que possam não só jogar sozinhos, mas também com outros colegas.

\section{Discussão}

Os resultados apontam que 0 PREVINIX foi validado pelos adolescentes, público alvo do jogo, como tecnologia educativa adequada para ações de educação em saúde com foco na prevenção, identificação e tratamento das IST's.

Inicialmente, verifica-se a efetividade do PREVINIX para o ganho de informações sobre as IST's, expresso na ampliação do número de acertos nas respostas ao questionário aplicado antes e após a ação educativa. Destaca-se que esta pesquisa permite afirmar que há ganho de informações imediatas, sendo necessários estudos posteriores que acompanhem a permanência destas informações bem como seu impacto para a adoção de comportamentos seguros na prática sexual a médio e longo prazo.

Ainda em relação ao ganho de informações sobre as IST's, observa-se que os adolescentes apresentaram um quantitativo maior de acertos no questionário aplicado antes da ação na temática sobre HIV/AIDS. Almeida, 
Bertuci ${ }^{16}$ explicam este fato apontando que campanhas de conscientização e propagandas de televisão são mais voltadas para este tema. No entanto, apesar dos adolescentes possuírem mais informações acerca da AIDS, estas podem ser equivocadas ou insuficientes, aumentando a exposição deste público às infecções ${ }^{1}$.

Concomitante a maior informação prévia sobre HIV e AIDS, observa-se o baixo nível de conhecimento em relação às outras doenças, especialmente a Sífilis ${ }^{17}$. Estas questões justificam a importância de estratégias educativas, como o PREVINIX, que invistam no diálogo com os adolescentes sobre esta temática.

Em relação aos objetivos e a relevância do jogo digital, o conteúdo analisado apontou que os propósitos do PREVINIX foram atendidos uma vez que os adolescentes puderam construir informações sobre as IST's juntamente com o mediador e identificaram a importância do tema no seu contexto de vida.

A literatura identifica que na adolescência os riscos epidemiológicos para IST's são maiores ${ }^{18}$. O início precoce da vida sexual torna os adolescentes mais vulneráveis, assim como o não uso dos métodos de prevenção ${ }^{18}$. Isso ocorre porque não basta apenas conhecer 0 preservativo, é preciso compreender a sua importância, a forma correta de sua utilização e os possíveis impactos da sua falta de uso ${ }^{19}$.
Neste sentido é importante abordar a prevenção na adolescência de forma clara para que seja efetiva junto ao público alvo $^{10,16,19}$.

Considerando estes aspectos, compreende-se que o PREVINIX pode ser utilizado como um recurso mediador da relação a ser estabelecida pelos adolescentes com o educador, de forma dialógica, e possibilitar a construção de um momento interativo, divertido e leve para abordar temas tabus e estimular a participação dessa população.

Destaca-se que os adolescentes sugerem que, para além da prevenção, sejam ampliadas as perguntas e discussões sobre como lidar com as IST's caso suspeitem de sua ocorrência. Neste sentido, serão incluídas no jogo mais questões sobre o tratamento e sobre as formas de acionamento das redes sociais de suporte que podem apoiar os adolescentes nestas situações.

O PREVINIX também proporcionou a reflexão dos adolescentes sobre os temas abordados e a ressonância destes nos seus contextos reais de vida. Essa congruência temática com os interesses dos adolescentes pode ter ocorrido, não somente pelo conteúdo ser baseado em aspectos destacados pelo Ministério da Saúde, mas ser, sobretudo, reflexo de questões ouvidas de adolescentes em experiências anteriores à pesquisa ${ }^{1,10,12}$. Neste sentido, destaca-se que a intencionalidade dos autores não era elaborar um jogo que se configurasse 
somente na compreensão dos "conceitos" sobre as IST's, mas que estas fossem abordadas de uma forma que possibilitasse que conhecimentos construídos possam ser utilizados pelos adolescentes na vida real.

Os adolescentes afirmaram no grupo focal que o PREVINIX pode ser uma ferramenta utilizada por profissionais de saúde e da educação. O ambiente escolar caracteriza-se como um cenário conveniente, que pode facilitar o vínculo entre os setores educação e saúde possibilitando 0 desenvolvimento de práticas de educação em saúde. O trabalho em equipe destes profissionais é uma estratégia de cuidado ao estudante/adolescente. O PREVINIX tem potencial para agir como facilitador na construção do vínculo entre o serviço de saúde e de educação com os adolescentes a fim de promover ações de educação em saúde voltada para este público.

No domínio Estrutura e Apresentação, os adolescentes indicaram que a linguagem do jogo está adequada e de fácil compreensão. Com relação às imagens utilizadas no jogo, os adolescentes expressaram reações como nojo, espanto e repulsa em olhar. Entretanto, quando questionados sobre sua relevância, afirmaram que as mesmas são de extrema importância, uma vez que trazem um conteúdo informativo indispensável para 0 jogo. Autores ${ }^{20}$ apontam que as imagens utilizadas em jogos digitais apresentam forte influência para a compreensão do conteúdo abordado, pois enriquecem o processo de aprendizagem. Assim, optou-se pela manutenção das imagens utilizadas por estas configurarem-se como ferramentas educativas importantes para que o jogo alcance os objetivos.

Os adolescentes relataram também que a tecnologia utilizada no jogo enquadra-se na faixa etária, corroborando estudos ${ }^{6,9,10}$ que asseguram que os jogos, por serem atividades comuns para os adolescentes, são um recurso lúdico importante para ser utilizado com essa faixa etária. Destaca-se que os jovens consideraram o jogo tão apropriado para o objetivo a que se propõe que pediram para que o mesmo fosse disponibilizado para uso em celular para que pudessem utilizálo em outros espaços.

Conforme também observado neste estudo, autores ${ }^{9}$ apontam que quando se usa o jogo digital no processo de aprendizagem é perceptível o envolvimento e a motivação que ele oferece, contribuindo para o planejamento de situações, formulação de hipóteses, tomadas de decisões e discussão de seus efeitos de forma individual ou grupal.

Ainda em relação à estrutura e apresentação do PREVINIX, os adolescentes apontaram a utilização das prendas como negativas para o processo do jogo. A intencionalidade das pesquisadoras foi, através de prendas, estimular a motivação para participação no jogo, sendo que nem sempre isto é 
necessário. Assim, na versão final será opcional o "pagamento" da prenda pelo grupo. Os adolescentes também sugeriram, e será acatado, a diminuição do tempo de resposta as questões a fim de dar maior dinamicidade ao jogo.

No que se refere a Vivência da experiência educativa, os adolescentes compreenderam que durante 0 jogo tiveram possibilidade de se expressar e colocar suas dúvidas com o auxílio do mediador. O mediador atua como agente potencializador e, portanto, essencial na construção do conhecimento a partir de uma metodologia dialógica que considera os saberes prévios dos envolvidos, respeita os projetos de vida dos e das adolescentes e acolhe as dúvidas sem opressão com vistas a promover o protagonismo dos e das adolescentes na construção do conhecimento ${ }^{14}$.

Considerando a importância do mediador para a efetividade do PREVINIX não será realizada uma versão do jogo para disponibilização para uso em smartphone. No entanto, aponta-se a intencionalidade de desenvolvimento de um outro jogo que seja disponibilizado aos jovens para uso em equipamentos celulares que se configure como uma continuidade do jogo PREVINIX, na qual os conhecimentos construídos coletivamente possam ser revisitados e rememorados.

\section{Conclusão}

Esta pesquisa apontou que 0 PREVINIX é uma tecnologia educativa válida para ser utilizada na promoção de saúde sexual e reprodutiva dos e das adolescentes, no que se refere a temática das IST's.

Observou-se que o jogo promoveu o ganho imediato de informações sobre as IST's, atingiu os objetivos, é considerado relevante pelos adolescentes, que também apontam a adequação de sua estrutura e apresentação. Além disso, o estudo indicou o uso do jogo digital como estratégia eficaz para a criação de um espaço de educação em pautada na construção conjunta entre os atores envolvidos.

A utilização do PREVINIX contribui para que o educador construa com os adolescentes um espaço divertido e com maior leveza para a discussão de assuntos muitas vezes considerados constrangedores tanto pelos jovens quanto pelos próprios profissionais.

Consideramos importante ressaltar que os profissionais que utilizarem 0 PREVINIX devem estar cientes não apenas dos conhecimentos específicos das IST's, mas também atentar-se à maneira de conduzir a ação educativa, considerando o diálogo como direcionador de suas atitudes. As observações realizadas durante a utilização do PREVINIX permitem afirmar que o alcance de sua efetividade não é proveniente da 
utilização do jogo apenas, mas depende, sobretudo, da intencionalidade do educador. Neste sentido, foi elaborado um material de apoio, que será disponibilizado junto com o jogo, no qual além de questões especificas sobre o funcionamento da tecnologia, serão abordados conteúdos referentes a construção do espaço educativo.

Esta pesquisa encerra-se sinalizando 0 potencial do desenvolvimento de estratégias que aproximem adolescentes e educadores na construção de espaços educativos nos quais ambos possam e se sintam desafiados a participarem ativamente, questionarem e construírem conhecimentos que sejam uteis e efetivos para subsidiar mudanças de atitudes e práticas no contexto da vida real.

Agradecimentos: Agradecemos ao CNPQ pelo apoio financeiro para a realização da pesquisa e à Puga Studios pela parceria no desenvolvimento do jogo.

\section{Referências}

1. Bechara AMD, Gontijo DT, Medeiros M, Facundes, VLD. "Na brincadeira a gente foi aprendendo": promoção de saúde sexual e reprodutiva com homens adolescentes. Revista Eletrônica de Enfermagem. 2013; 15(1): 25-33. 2- Brasil. Ministério da Saúde. Secretaria de Atenção à Saúde. Departamento de Ações Programáticas e Estratégicas. Proteger e cuidar da saúde de adolescentes na atenção básica. Brasília: Ministério da Saúde, 2017.

3. Brasil. Instituto Brasileiro de Geografia e Estatística. Pesquisa Nacional de Saúde do Escolar 2016. Rio de Janeiro: IBGE; 2016.

4. Bordignon MNFD, Liberali $R$, Bordignon JCP. Causas da não utilização de preservativos nas práticas sexuais de adolescentes: revisão integrativa. Revista de enfermagem UFPE online. 2017; 11(1): 207-213.

5.Brasil. Ministério da Saúde. Boletim epidemiológico AIDS/DST. Brasília: Ministério da Saúde; 2016.

6. Oliveira RNG, Gessner R, Souza VD, Fonseca RMGC. Limites e possibilidades de um jogo online para a construção de conhecimento de adolescentes sobre a sexualidade. Ciência \& Saúde Coletiva. 2016; 21 (8): 2383-2392.

7. Souza V, Gazzinelli MF, Soares AN, Fernandes MM, Oliveira RNG, Fonseca RMGS. The game as strategy for approach to sexuality with adolescents: theoretical-methodological reflections. RevBrasEnferm [Internet]. 2017;70(2):376-83.

8. Shegog R, Brown K, Bull S, Chistensen JL, Hieftje K, Jozkowski KN, Ybarra ML. Serious games for Sexual Health. Games for Health Journal. 2015; 4(2): 69-77.

9. Desmet A, Shegog R, Ryckeghen DV, Crombez G, Bourdeaudhuij IA. Systematic Review and Meta-analysis of Interventions for Sexual Health Promotion Involving Serious Digital Games. GAMES FOR HEALTH JOURNAL: Research, Development, and Clinical Applications. 2015; 4(2): 78-90.

10. Monteiro RJS, Oliveira MPCA, Belian RB, Lima LS, Santiago ME, Gontijo DT. DECIDIX: Encontro da Pedagogia Paulo Freire com os serious games no campo da educação em saúde com adolescentes. Ciênc. saúde coletiva. 2018; 23( 9 ): 2951-2962.. 11- Braz, LCSB. Percepções de adolescentes escolares acerca de uma ação educativa para promoção da saúde sexual [Dissertação]. Natal: Universidade Federal do Rio Grande do Norte - UFRN; 2019.

12. Gontijo DT, Vasconcelos ACS, Monteiro RJS, Facundes VLD, Trajano MFC, Lima LS. Occupational Therapy and Sexual and Reproductive Health Promotion in Adolescence: A Case Study. 
Occupational Therapy International. 2015;

22: 19-28.

14. Freire P. Pedagogia do Oprimido. Rio e Janeiro: Paz e Terra; 2015.

15. Gonçalves MS, Celedônio RF, Targino

$\mathrm{MB}$, Albuquerque TO, Flauzino PA,

Bezerra AN, et al. Construção e

validação de cartilha educativa para

promoção da alimentação saudável entre

pacientes diabéticos. Rev Bras Promoç

Saúde. 2019;32:7781

16. Almeida A, Bertucci TCSC. O

conhecimento dos adolescentes sobre as

doenças sexualmente transmissíveis e

métodos de prevenção: um estudo de

caso em uma escola pública estadual no

município de Antônio João- MS. Caderno

Magsul de Ciências Biológicas. 2017;

4(1): 6 .

17. BRASIL. Ministério da Saúde. Boletim Epidemiológico Sífilis. Brasília: Ministério da Saúde; 2017.

18. Costa RSL, Silva WB, Nascimento

KJO. Percepção de risco de adolescentes escolares em relação às infecções

sexualmente transmissíveis em duas

escolas de ensino médio do

Acre. Ciência em Foco. 2018; 2(2): 59-

72.

19. Scopacasa LF. Validação de jogo educativo para construção do

conhecimento de adolescentes acerca da prevenção de DST/Aids [Dissertação].

Fortaleza: Universidade Federal do Ceará - UFC; 2013.

20. Ramos DK, Martins PN, Anastácio BS. $A$ função da narrativa e dos personagens em um jogo digital educativo: análise do jogo Saga dos Conselhos. Interfaces

Científicas-Educação. 2017; 6(1): 59-70. 


\section{Anexos}

Quadro1: Percentual Global e por temas de resposta dos adolescentes antes e após o uso do jogo digital

\begin{tabular}{|c|c|c|c|c|c|c|c|c|c|c|c|c|c|c|c|c|}
\hline & \multicolumn{4}{|c|}{ Certo } & \multicolumn{4}{|c|}{ Errado } & \multicolumn{4}{|c|}{ Não sei } & \multicolumn{4}{|c|}{ Ignorado } \\
\hline & \multicolumn{2}{|c|}{ Antes } & \multicolumn{2}{|c|}{ Depois } & \multicolumn{2}{|c|}{ Antes } & \multicolumn{2}{|c|}{ Depois } & \multicolumn{2}{|c|}{ Antes } & \multicolumn{2}{|c|}{ Depois } & \multicolumn{2}{|c|}{ Antes } & \multicolumn{2}{|c|}{ Depois } \\
\hline Temática & $\mathrm{N}$ & $\%$ & $\mathrm{~N}$ & $\%$ & $\mathrm{~N}$ & $\%$ & $\mathrm{~N}$ & $\%$ & $\mathrm{~N}$ & $\%$ & $\mathrm{~N}$ & $\%$ & $\mathrm{~N}$ & $\%$ & $\mathrm{n}$ & $\%$ \\
\hline HIV/AIDS & 69 & 41,1 & 120 & 71,4 & 52 & 31,0 & 36 & 21,4 & 46 & 27,4 & 12 & 7,1 & 1 & 0,6 & 0 & 0,0 \\
\hline Sífilis & 69 & 24,1 & 204 & 72,9 & 23 & 8,2 & 50 & 17,9 & 185 & 66,1 & 26 & 9,3 & 3 & 1,1 & 0 & 0,0 \\
\hline Prevenção & 170 & 33,7 & 317 & 62,9 & 117 & 23,2 & 150 & 29,8 & 211 & 41,9 & 31 & 6,2 & 6 & 1,2 & 6 & 1,2 \\
\hline HPV & 96 & 42,9 & 146 & 65,2 & 27 & 12,1 & 60 & 26,8 & 98 & 43,8 & 18 & 8,0 & 3 & 1,3 & 0 & 0,0 \\
\hline Hepatite & 44 & 39,3 & 71 & 63,4 & 7 & 6,3 & 15 & 13,4 & 58 & 51,8 & 26 & 23,2 & 3 & 2,7 & 0 & 0,0 \\
\hline Outras & 29 & 25,9 & 102 & 91,1 & 8 & 7,1 & 6 & 5,4 & 73 & 65,2 & 4 & 3,6 & 2 & 1,8 & 0 & 0,0 \\
\hline $\begin{array}{l}\text { Todas as } \\
\text { temáticas }\end{array}$ & 477 & 34,1 & 960 & 68,6 & 234 & 16,7 & 317 & 22,6 & 671 & 47,9 & 117 & 8,4 & 18 & 1,3 & 6 & 0,4 \\
\hline
\end{tabular}

Fonte:

Quadro2: - Índice de Concordância em relação ao domínio objetivo

\begin{tabular}{|c|c|c|c|c|c|c|c|}
\hline Objetivo do jogo & CT & $\mathrm{CP}$ & NCND & $\mathrm{DP}$ & DT & NM & IVC \\
\hline $\begin{array}{l}\text { 1- O jogo permite com que você pense sobre os sinais e } \\
\text { sintomas que as IST's podem apresentar }\end{array}$ & 50 & 3 & 3 & 0 & 0 & 0 & 0,94 \\
\hline $\begin{array}{l}\text { 2- O jogo faz com que você pense sobre as causas das } \\
\text { IST's }\end{array}$ & 43 & 10 & 3 & 0 & 0 & 0 & 0,94 \\
\hline 3- O jogo traz formas de tratamentos para as infecções & 45 & 5 & 5 & 0 & 0 & 1 & 0,89 \\
\hline $\begin{array}{l}\text { 4- O jogo traz orientações que você considera } \\
\text { importantes em relação à saúde sexual }\end{array}$ & 53 & 1 & 1 & 1 & 0 & 0 & 0,96 \\
\hline
\end{tabular}

Legenda: CT: Concordo Totalmente; CP: Concordo Parcialmente; NCND: Nem Concordo Nem Discordo; DP:

Discordo Parcialmente; DT: Discordo Totalmente; NM: Não marcado.

Quadro3: - Índice de Concordância quanto ao domínio Relevância

\begin{tabular}{|c|c|c|c|c|c|c|c|}
\hline Relevância do jogo & CT & $\mathrm{CP}$ & NCND & $\mathrm{DP}$ & DT & NM & IVC \\
\hline 1- O tema do jogo é relevante para o adolescente & 51 & 1 & 3 & 1 & 0 & 0 & 0,92 \\
\hline $\begin{array}{l}\text { 2- O jogo permite que você aprenda sobre prevenção e } \\
\text { tratamento das IST's }\end{array}$ & 50 & 3 & 3 & 0 & 0 & 0 & 0,94 \\
\hline $\begin{array}{l}\text { 3- O jogo permite que você se sinta mais capacitado } \\
\text { e consciente em relação à IST }\end{array}$ & 45 & 7 & 2 & 0 & 1 & 1 & 0,92 \\
\hline $\begin{array}{l}\text { 4- O jogo permite que você leve o que aprendeu para sua } \\
\text { vida }\end{array}$ & 51 & 4 & 0 & 0 & 0 & 1 & 0,98 \\
\hline 5- O jogo é importante para ser usado com adolescentes & 50 & 2 & 1 & 0 & 0 & 3 & 0,92 \\
\hline
\end{tabular}

CT: Concordo Totalmente; CP: Concordo Parcialmente; NCND: Nem Concordo Nem Discordo; DP: Discordo

Parcialmente; DT: Discordo Totalmente; NM: Não marcado.

Quadro 4: - Índice de Validade de Concordância quanto ao domínio Estrutura e apresentação

\begin{tabular}{|c|c|c|c|c|c|c|c|}
\hline Estrutura e apresentação do jogo & CT & $\mathrm{CP}$ & NCND & $\mathrm{DP}$ & DT & NM & IVC \\
\hline 1- As perguntas do jogo estão de fácil compreensão & 41 & 11 & 3 & 0 & 0 & 1 & 0,92 \\
\hline 2- O jogo está adequado a idade de adolescente & 47 & 5 & 3 & 0 & 0 & 1 & 0,92 \\
\hline $\begin{array}{l}\text { 3- A forma do jogo é atrativo e agradável para } \\
\text { os adolescentes }\end{array}$ & 47 & 6 & 2 & 0 & 0 & 1 & 0,94 \\
\hline 4- O jogo está de acordo com a sua realidade & 48 & 1 & 3 & 1 & 0 & 3 & 0,87 \\
\hline
\end{tabular}

Legenda: CT: Concordo Totalmente; CP: Concordo Parcialmente; NCND: Nem Concordo Nem Discordo; DP:

Discordo Parcialmente; DT: Discordo Totalmente; NM: Não marcado. 


\section{Como citar este artigo}

Oliveira ARPS; Valério DOS; Rosas MA; Azevedo LN; Silva BN; Gontijo DT. Validação do jogo digital PREVINIX para prevenção de Infecções Sexualmente Transmissíveis na adolescência. Revista de Saúde Digital e Tecnologias Educacionais. [online], volume 4, n. 2. Editor responsável: Luiz Roberto de Oliveira. Fortaleza, mês e ano, p.163-178. Disponível em: http://periodicos.ufc.br/resdite/index. Acesso em "dia/mês/ano".

Data de recebimento do artigo: 26/09/2019

Data de aprovação do artigo: 27/11/2019 